\title{
Design of a Coiled-Coil-based Model Peptide System to Explore the Fundamentals of Amyloid Fibril Formation
}

\author{
Michel O. Steinmetz, ${ }^{1,4}$ Carlos García-Echeverría, ${ }^{2}$ and Richard A. Kammerer ${ }^{3,4}$
}

(Accepted September 25, 2004)

\begin{abstract}
Protein deposition as amyloid fibrils underlies more than twenty severely debilitating human disorders. Interestingly, recent studies suggest that all peptides and proteins possess an intrinsic ability to assemble into amyloid fibrils similar to those observed in disease states. The common properties and characteristics of amyloid aggregates thus offer the prospect that simple model systems can be used to systematically assess the factors that predispose a native protein to form amyloid fibrils and understand the origin and progression of fatal disorders associated with amyloid formation. Here, we report the de novo design of a 17-residue peptide model system, referred to as $\operatorname{cc} \beta$, which forms a protein-like coiled-coil structure under ambient solution conditions but can be easily converted into amyloid fibrils by raising the temperature. Oxidation of methionine residues at selected hydrophobic positions completely abolished amyloid fibril formation of the peptide while not interfering with its coiled-coil structure. This finding indicates that a small number of site-specific hydrophobic interactions can play a major role in the packing of polypeptide chain segments within amyloid fibrils. The simplicity and characteristics of the $\operatorname{cc} \beta$ system make it highly suitable for probing molecular details of the assembly of amyloid structures.
\end{abstract}

KEY WORDS: Coiled coil; de novo design; amyloid.

\section{INTRODUCTION}

The deposition of proteins in the form of amyloid fibrils and plaques is the characteristic feature of more than twenty degenerative conditions affecting either the central nervous system or a variety of peripheral

\footnotetext{
${ }^{1}$ Biomolecular Research, Structural Biology, Paul Scherrer Institut, CH-5232 Villigen PSI, Switzerland; Tel: +41-56-310-4754; Fax: +41-56-310-5288; e-mail: michel.steinmetz@psi.ch

${ }^{2}$ Novartis Institutes for BioMedical Research, CH-4002 Basel, Switzerland.

${ }^{3}$ Wellcome Trust Centre for Cell-Matrix Research, School of life Sciences, University of Manchester, Michael Smith Building, Oxford Road, Manchester, M13 9PT, UK; Tel: +44-161-2751504/7513; Fax: +44-161-275-1505; e-mail: richard.kammerer@ manchester.ac.uk

${ }^{4}$ Correspondence should be addressed to: Michel O. Steinmetz and Richard A. Kammerer.
}

tissues (Lansbury 1999; Perutz 1999; Prusiner 2001; Sacchettini and Kelly 2002; Taylor et al. 2002; Aguzzi and Haass 2003; Stefani and Dobson 2003). These conditions include Alzheimer's, Parkinson's and the prion diseases, several forms of fatal systemic amyloidosis, and at least one condition associated with medical intervention (haemodialysis). Due to the severity of these diseases, they have an enormous

\footnotetext{
Abbreviations: CD, circular dichroism; Fmoc, 9-fluorenylmethoxycarbonyl; EDT, 1,2-ethanedithiol; GRAVY, Grand average hydrophaty; HPLC, high-performance liquid chromatography; MALDI-TOF, matrix-assisted laser-desorption ionization time-offlight mass spectroscopy; MBHA-resin, 4-methylbenzhydrylamineresin; PAL, tris(alkoxy)benzylamide linker; PBS, phosphate buffered saline; PEG, polyethylene glycol; TEM, transmission electron microscope; TPTU, O-(1,2-dihydro-2-oxo-pyridyl)- $N, \mathrm{~N}$, $\mathrm{N}^{\prime}, \mathrm{N}^{\prime}$-tetramethyluronium tetrafluoroborate; TFA, trifluoroacetic acid; UV, ultraviolet.
} 
impact to our present-day human health and welfare. The detailed structural characterization of amyloid fibrils and the systematic assessment of factors that predispose a native protein to misfold into amyloid aggregates are therefore indispensable for understanding the origin and progression of the diseases. Consistent with the propensity of seemingly unrelated proteins to polymerize in vivo, recent studies suggest that many peptides and proteins not associated with amyloid diseases possess an intrinsic ability to assemble into amyloid fibrils similar to those observed in disease states under appropriate conditions (Chiti et al. 1999; Fandrich et al. 2001). Furthermore, it has been suggested that amyloid fibril formation is an evolutionary conserved biological pathway used to generate natural product nanostructures (Kelly and Balch 2003). As a result, amyloid fibrils have attracted much attention for nanotechnological and material science applications (Zhang 2003).

Interestingly, all amyloid fibrils share common characteristics. A key feature of all amyloid fibrils is their apparently similar molecular architecture. Electron micrographs of amyloid deposits from sequentially or structurally unrelated peptides and proteins frequently show the presence of uniform and unbranched fibrils with a diameter of 7 to $12 \mathrm{~nm}$. These fibrils are often several micrometers in length and are usually composed of many protofilaments (Serpell et al. 1997). The internal structure of amyloid fibrils is defined by their characteristic X-ray fibre diffraction patterns that are generally interpreted as a "cross- $\beta$ ", structure. In this type of structure, the polypeptide chain is organized in laminated layers of $\beta$-sheets arranged parallel to the fibre axis with their constituent $\beta$-strands running approximately perpendicular to the long fibril axis (Sunde et al. 1997). Together with the requirement of a nucleation-dependent step for productive assembly (Rochet and Lansbury 2000), these findings strongly suggest that amyloid fibrils are formed by a common self-assembly pathway.

Although in recent years substantial progress has been made in our understanding of the overall characteristics of amyloid structures and their formation, detailed knowledge of the intra- and inter-molecular interactions that promote and stabilize these highly organized assemblies is still lacking. Furthermore, the molecular details underlying the process of amyloid formation are still poorly understood. These gaps in our knowledge result from the non-crystalline nature of amyloid fibrils, which makes their high-resolution structural analysis extremely challenging, and from the complexity and diversity of the different proteins that form amyloid aggregates. It is generally acknowledged that one major factor for driving fibril formation is the generic property of the polypeptide backbone to form hydrogen bonds. A number of studies indicate, however, that the amino acid sequence can profoundly influence the propensity of a polypeptide chain to form amyloid fibrils. In such cases, the effects of limited sequence changes can be rationalized in terms of perturbations in the various native or nonnative protein conformations (Booth et al. 1997; Ramirez-Alvarado et al. 2000, Villegas et al. 2000). In the case of globular proteins, at least partial unfolding is an essential step in the conversion to amyloid fibrils. Thus, destabilization of the native state of natural proteins has been reported to be directly coupled to the propensity to form amyloid deposits and is generally accepted as a major factor controlling amyloid formation (Hurle et al. 1994; McCutchen et al. 1995; Booth et al. 1997; Chiti et al. 2000; Ramirez-Alvarado et al. 2000; Zhu et al. 2003). It is well established, however, that changes in the hydrophobicity, charge, secondary structure propensity, and residual $\alpha$-helical structure in the unfolded state, occurring as a result of mutations, can influence the probability of amyloid fibril formation (Nielsen et al. 2001; Chiti et al. 2002; Hammarstrom et al. 2002; Lopez De La et al. 2002; Tjernberg et al. 2002a; Burke et al. 2003; Chiti et al. 2003; Smith et al. 2003). In some cases, it appears that mutations within distinct polypeptide chain segments can promote fibril formation (Nielsen et al. 2001, Ciani et al. 2002) suggesting that specific regions are of particular importance in the nucleation or stabilization of the amyloid structure. Juxtaposition of hydrophobic and/or polar/charged sequence segments may play a particularly important role in the organization of the $\beta$-sheets within the protofilament (Perutz et al. 1994; Zhang and Rich 1997; West et al. 1999; Tjernberg et al. 1999; Takahashi et al. 2000; Balbirnie et al. 2001). Together, these findings suggest that both long- and short-range interactions within a distinct sequence context can participate in the process of amyloid formation.

The common properties and characteristics of amyloid aggregates offer the prospect that simple model systems can be used to systematically assess the factors that predispose a native protein to form amyloid fibrils and understand the origin and progression of fatal disorders associated with amyloid formation. We report here a simplified peptide system, referred to as $\operatorname{cc} \beta$, successfully designed de novo to fold into a $\alpha$-helical coiled-coil conformation 
under ambient conditions, but to transform into amyloid fibrils at elevated temperatures. We demonstrate that its simplicity and characteristics make the $\operatorname{cc} \beta$ system highly suitable for probing molecular details of the assembly of amyloid structures.

\section{MATERIALS AND METHODS}

\section{Peptide Synthesis and Derivatization}

Chain assembly was carried out on a Milligen 9050 automated peptide synthesizer (continuous flow), starting with an Fmoc-PAL-PEG-MBHA resin for establishing the C-terminal carboxamide (Albericio et al. 1990), and using chemical protocols based on Fmoc chemistry. The required Fmoc amino acids (3 equiv.) were incorporated using their 2,4,5-trichlorophenyl esters with minimum reaction times of $30 \mathrm{~min}$. Side chains were protected with the following groups: tert-butyl for serine and glutamic acid; 2,2,5,7,8-pentamethyl-chroman-6-sulfonyl for arginine. We recommend double coupling for the incorporation of arginine-3,8,10 and 15, isoleucine- 2 and 9, leucine-5, and serine-1. These Fmoc-amino acids ( 3 equiv.) were incorporated using TPTU (3 equiv.) as coupling agent in the presence of diisopropylamine (6 equiv.). The completed peptide resins were simultaneously deprotected and cleaved by treatment with TFA: $\mathrm{H}_{2} \mathrm{O}(95: 5, \mathrm{v} / \mathrm{v}$; for $\mathrm{cc} \beta-\mathrm{p})$ or TFA: $\mathrm{H}_{2} \mathrm{O}$ :EDT $(76: 4: 20, \mathrm{v} / \mathrm{v} /$ $\mathrm{v}$; for $\operatorname{cc} \beta$-Met) for $3 \mathrm{~h}$ at room temperature. The filtrate from each cleavage reaction was precipitated in diisopropyl etherpetroleum ether $\left(1: 1, \mathrm{v} / \mathrm{v}\right.$; at $\left.0^{\circ} \mathrm{C}\right)$, and the precipitate was collected by filtration. The crude peptides were purified by mediumpressure liquid chromatography using a Merck $\mathrm{C}_{18}$-column eluted with an acetonitrile-water gradient containing $0.1 \%$ TFA.

Controlled oxidation of methionines in cc $\beta$-Met was performed as follows (García-Echeverría 1996): $70 \mathrm{mg}$ of purified cc $\beta$-Met were dissolved in $6.3 \mathrm{ml}$ of $\mathrm{MeCN}: \mathrm{H}_{2} \mathrm{O}(1: 1, \mathrm{v} / \mathrm{v})$ and $0.7 \mathrm{ml}$ of $\mathrm{H}_{2} \mathrm{O}_{2}-30 \%$ were added. The progress of the reaction was monitored by analytical HPLC and MALDI-TOF analyses. After full conversion of cc $\beta$-Met to the target peptide, the reaction was quenched by addition of ascorbic acid and the crude mixture was purified by medium-pressure liquid chromatography as described above. The purity of the final peptide was verified by reversed-phase analytical HPLC on a Chromolith Speed ROD RP18e column $(50 \times 4.6 \mathrm{~mm})$ : linear gradient over $2.5 \mathrm{~min}$ of $\mathrm{MeCN} / 0.09 \%$ TFA and $\mathrm{H}_{2} \mathrm{O} / 0.1 \%$ TFA from $6: 44$ to $7: 3 \mathrm{v} / \mathrm{v}$; flow rate $3.0 \mathrm{ml} / \mathrm{min}$, detection at $215 \mathrm{~nm}$. Single peaks were observed at following retention times $t_{\mathrm{R}}=2.03 \mathrm{~min}, 2.01 \mathrm{~min}$, and $1.97 \mathrm{~min}$ for $\operatorname{cc} \beta-\mathrm{p}, \operatorname{cc} \beta$-Met, and $\operatorname{cc} \beta-\mathrm{MetO}$, respectively. MALDITOF mass spectrometric analyses revealed molecular masses within $0.1 \%$ of the expected values (negative-ion mode): 2092.7 (calc. 2093.5, сc $\beta-p$ ), 2171.7 (calc. 2171.6, cc $\beta-M e t$ ) and 2203.6 (calc. 2203.6, cc $\beta$-MetO). Quantitative amino acid analyses (Beckman 6300 amino acid analyzer) of the purified peptides after acid hydrolyzes gave the expected composition.

\section{Circular Dichroism Spectroscopy}

Peptide samples were analyzed in $10 \mathrm{mM}$ sodium phosphate buffer (pH 7.4) containing $150 \mathrm{mM} \mathrm{NaCl}$ (PBS). Far-ultraviolet (UV) circular dichroism (CD) spectra and urea unfolding profiles were recorded on a Jasco J-720 spectropolarimeter (Jasco Inc.) equipped with a temperature controlled quartz cell of $0.1 \mathrm{~cm}$ pathlength. The recorded spectra were evaluated with the Jasco software package. The midpoints of the urea-induced equilibrium transitions, $C_{\mathrm{m}}$ 's, and thermal melts, $T_{\mathrm{m}}$ 's, were taken as the maximum of the derivative $\mathrm{d}$ (Fraction transformed)/d[urea] and $\mathrm{d}[\Theta]_{222} / \mathrm{dT}$, respectively.

\section{Analytical Ultracentrifugation}

Analytical ultracentrifugation (AUC) was performed at $5^{\circ} \mathrm{C}$ on an Optima XL-A analytical ultracentrifuge (Beckman Instruments) equipped with an An-60Ti rotor. The peptides were analyzed in PBS and peptide concentrations were adjusted to 0.1$0.5 \mathrm{mg} / \mathrm{ml}$. Sedimentation equilibrium experiments were performed between 40,000 and 50,000 rpm. The partial specific volumes of the peptides were calculated from their amino-acid sequence. Solvent density for PBS was taken as $1.005 \mathrm{~g} / \mathrm{ml}$.

\section{Negative Staining and Transmission Electron Microscopy}

For negative staining, sample aliquots of $5 \mu \mathrm{l}$ were applied to a weakly glow-discharged carbon coated 400 -mesh/inch copper grid. The sample was allowed to adsorb for $30 \mathrm{sec}$, washed twice with water, and negatively stained for $20 \mathrm{sec}$ with $2 \%$ (w/v) uranyl acetate. Specimens were examined in a Philips Morgagni transmission electron microscopy (TEM) operated at $80 \mathrm{kV}$. Micrographs were recorded with a Megaview III CCD camera at a nominal magnification of $30,000 \times$ and $50,000 \times$.

\section{Congo Red Staining}

The method of Janek et al. (Janek et al. 1999) was used with slight modifications. Briefly, a saturated solution of Congo red (Aldrich) was prepared in $80 \%$ ethanol. 10-20 $\mu \mathrm{l}$ of a concentrated filamentous peptide preparation was placed on a glass slide, air dried and incubated for $10 \mathrm{~min}$ with the Congo red stock solution. After being washed with water and ethanol, the stained samples were examined under the microscope with bright and polarized light.

\section{RESULTS AND DISCUSSION}

\section{Design Rationale}

De novo protein design provides a powerful tool to elucidate and validate fundamental principles of protein structure and folding (DeGrado et al. 1999). The design of a preferably short and simple peptide sequence that adopts a native-like fold while displaying conformational interconversion (e.g., from $\alpha$-helix to $\beta$-sheet) and fibril formation should be beneficial for exploring the mechanistic details of amyloid formation. For the purpose of this study, we selected the $\alpha$-helical coiled-coil as a platform for the 
design. The $\alpha$-helical coiled-coil is the most widespread subunit oligomerization motif found in proteins (Lupas 1996; Kammerer 1997; Burkhard et al. 2001). This abundance reflects the importance and involvement of the motif in a large number of biological processes. Coiled coils consist of two to five amphipathic right-handed $\alpha$-helices that "coil" around each other in a slight supertwist. The sequences of left-handed coiled-coils are characterized by a heptad repeat of seven residues denoted a to $\mathbf{g}$ with a 3,4-hydrophobic repeat of mostly apolar amino acids at positions $\mathbf{a}$ and $\mathbf{d}$. Interactions between the core residues $\mathbf{a}$ and $\mathbf{d}$ and its two flanking positions e and $\mathbf{g}$ determine the number of strands, the parallel or antiparallel orientation of $\alpha$-helices, and the homo- or heterotypic association of subunits into a coiled-coil (Cohen and Parry 1990; Lupas 1996; Kohn and Hodges 1998).

Because of its simplicity, the coiled-coil structural motif has been extensively studied and used as a model system for de novo protein design (Kohn and Hodges 1998; DeGrado et al. 1999). Accordingly, the sequence-to-structure relationship of coiled-coils is well established. It has been shown that peptides comprising 15 to 21 residues can form stable coiled-coil structures ( $\mathrm{Su}$ et al. 1994; Burkhard et al. 2000), whereas the sequence length of a $\beta$-strand in an amyloid fibril is in the range of 5 to 26 residues (Benzinger et al. 1998; Tenidis et al. 2000). Based on the concept of coiled-coil trigger sequences (Kammerer et al. 1998; Steinmetz et al. 1998; Frank et al. 2000; Kammerer et al. 2001) and in combination with available information on peptides that undergo structural transitions (Zhang and Rich 1997; West et al. 1999; Takahashi et al. 2000; Ciani et al. 2002), we designed de novo a synthetic 17-residue peptide system, referred to as $\operatorname{cc} \beta$, with the potential to fold into both a stable $\alpha$-helical coiled-coil and a $\beta$-sheet type of structure (Fig. 1).

To keep the design as simple as possible, the number of different amino acids in the peptide was limited to a minimum of seven. Amino acids with high propensities for both the $\alpha$-helix and $\beta$-strand structure were chosen (Minor and Kim 1994; Chakrabartty and Baldwin 1995). To guarantee formation of a stable and specific two-stranded parallel coiled-coil structure, hydrophobic Ile and Leu residues were placed at the a and $\mathbf{d}$ positions of the two heptad repeats, respectively (Harbury et al. 1993). Positions $\mathbf{b}, \mathbf{c}, \mathbf{e}$, and $\mathbf{g}$ were occupied with Arg and Glu residues in a manner to allow for $\mathrm{i}$ to $\mathrm{i}+3$ and $\mathrm{i}$ to $i+4$ intrahelical and $i$ to $i^{\prime}+5$ (i.e., between the heptad $\mathbf{e}$ and $\mathbf{g}^{\prime}$ positions that flank the hydrophobic $\mathbf{a}$ and $\mathbf{d}$ core positions; Fig. 1b) interhelical attractive electrostatic interactions throughout the sequence (Fig. 1b, dashed curved lines). Such attractive electrostatic interactions are known to stabilize and direct parallel in-register chain orientation of coiledcoil structures (Kohn and Hodges 1998). No putative repulsive electrostatic interactions were allowed in the design. The core sequence was flanked by $\mathrm{N}$ - and $\mathrm{C}$ terminal acetylated Ser and amidated Gly capping residues (Richardson and Richardson 1998), respectively, that are expected to stabilize further the coiledcoil structure (Lu et al. 1999).

Amyloid fibrils are cross- $\beta$ structures organized in laminated layers of $\beta$-sheets that are arranged parallel to the fibre axis (Sunde et al. 1997). Thus, for sequences to self-assemble into amyloid aggregates they must contain the information to form multimeric $\beta$-sheets. Binary sequence patterning of hydrophobic and polar residues was found to strongly favor $\beta$-sheet and amyloid formation of designed peptides (Zhang and Rich 1997; West et al. 1999). This alternating pattern matches the structural repeat of $\beta$-strands with successive side chains pointing up and down throughout the sequence (Fig. 1c). Thus, such sequence elements are predisposed to form amphipathic $\beta$-strands that bury their hydrophobic faces by aggregating into large $\beta$-sheet and cross- $\beta$ structures (Hecht et al. 2004). In order to create two short seven and six residue long binary hydrophobic/polar sequence patterns in the $\operatorname{cc} \beta$ sequence platform, hydrophobic Ala and Leu residues were placed at the remaining $\mathbf{f} 7$ and $\mathbf{f} 14$ positions (Fig. 1a). As a result, the parent peptide, referred to as $\operatorname{cc} \beta-p$, has the potential for establishing complementary inter-chain interactions between hydrophobic and polar residues in both $\alpha$-helical coiled-coil (Fig. 1b) and $\beta$-sheet (Fig. 1c) assemblies.

To test the hypothesis that specific hydrophobic packing interactions play a crucial role in driving $\operatorname{cc} \beta$ amyloid formation, a peptide variant was prepared in which Met residues were placed at the heptad $\mathbf{f} 7$ and f14 positions, referred to as $\operatorname{cc} \beta$-Met. Mutations of heptad f residues are not expected to interfere with the coiled-coil structure as these positions do not participate in the formation of the hydrophobic core (Fig. 1b). Methionine was chosen for three reasons. First, methionine does not show a dramatically different intrinsic propensity to favor either $\alpha$-helix or $\beta$ sheet structures when compared to Ala or Leu (Minor and Kim 1994; Chakrabartty and Baldwin 1995). Thus no major conformational effect on the 


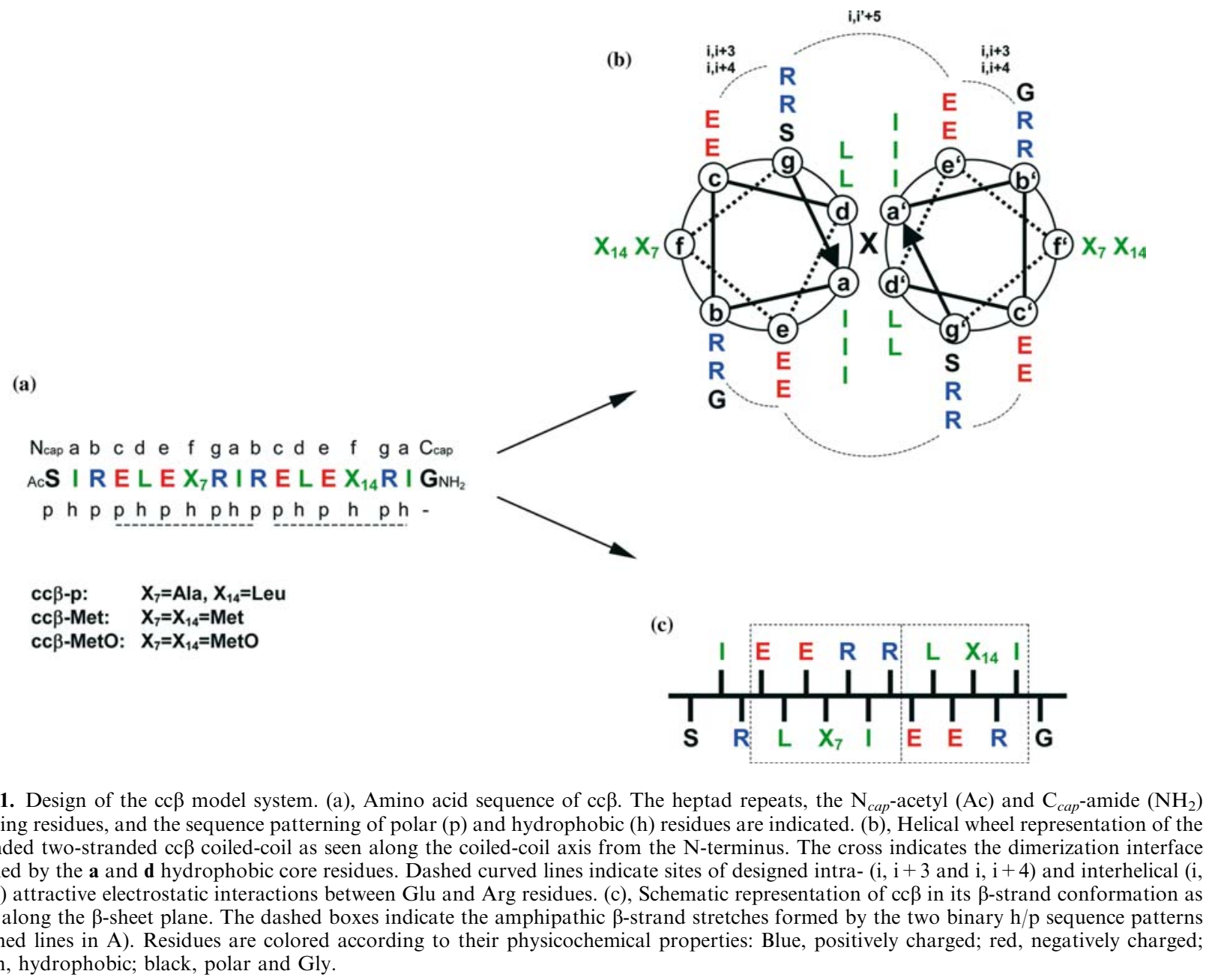

denatured state is expected. Second, the two methionine residues do not substantially alter the overall hydrophobicity of the $\operatorname{cc} \beta$ peptide variant: a difference of only 0.11 in the Grand average hydrophaty (GRAVY; (Kyte and Doolittle 1982)) index scores is calculated between $\operatorname{cc} \beta-p$ and $\operatorname{cc} \beta$-Met. Finally, the methionine side chain can be chemically oxidized to a polar sulfoxide group. The introduction of one single oxygen atom switches the character of the residue from hydrophobic to polar and, as a consequence of this change, disrupts the two short binary hydrophobic/polar sequence patterns present in the $\operatorname{cc} \beta$ sequence (Fig. 1c). This form of the peptide is referred to as $\operatorname{cc} \beta$-MetO.

The use of methionine in this work expands the "switchable residue" concept of Dado and Gellman, who used methionine residues to control the secondary structure propensity (i.e., $\alpha$-helix versus $\beta$-sheet) of an 18-mer peptide (Dado and Gellman
1993). The oxidation state of methionine has also been used to control the folding preferences of leucine-zipper peptides (García-Echeverría 1996).

\section{Design Validation}

The validity of the design was thoroughly challenged by various biophysical methods. The $\alpha$-helical coiled-coil state of the three peptides at $4^{\circ} \mathrm{C}$ was probed by CD spectroscopy. The far-UV CD spectra recorded at a concentration of $0.2 \mathrm{mg} / \mathrm{ml}$ confirmed substantial $\alpha$-helical structure for the peptides with well-defined minima at 208 and $222 \mathrm{~nm}$ (Fig. 2a). Sedimentation equilibrium studies under similar conditions yielded averaged molecular masses of 5.9, 6.3, and $6.2 \mathrm{kDa}$ for cс $\beta$-p, сc $\beta$-Met, and $\operatorname{cc} \beta$-MetO, respectively. These values are consistent with the formation of trimeric structures (the sequence derived molecular masses of 
(a)

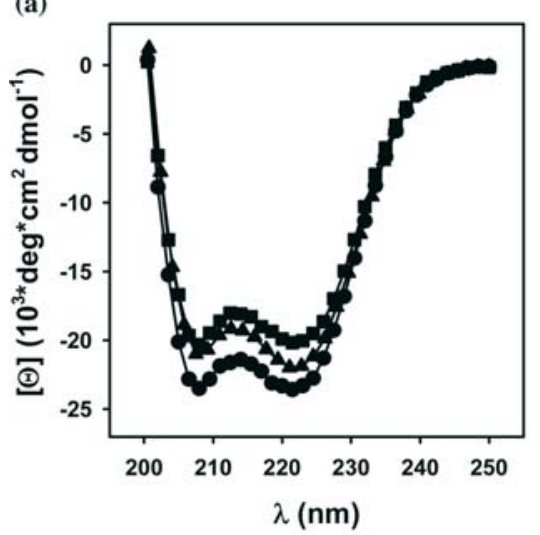

(c)
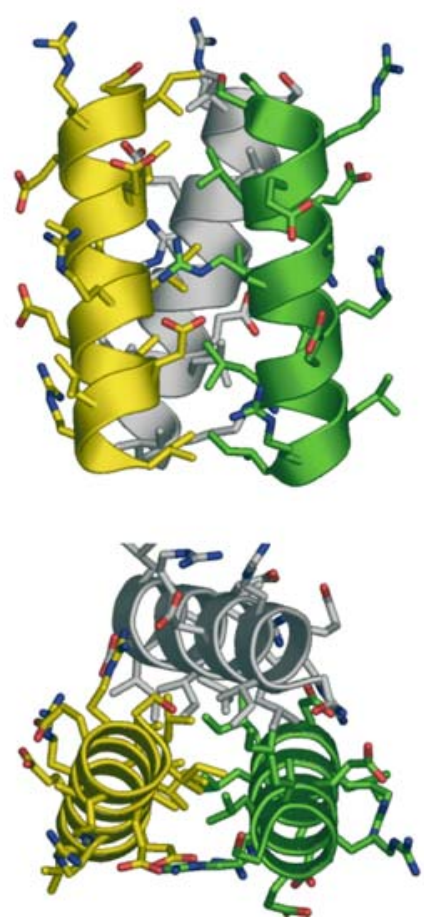

(b)
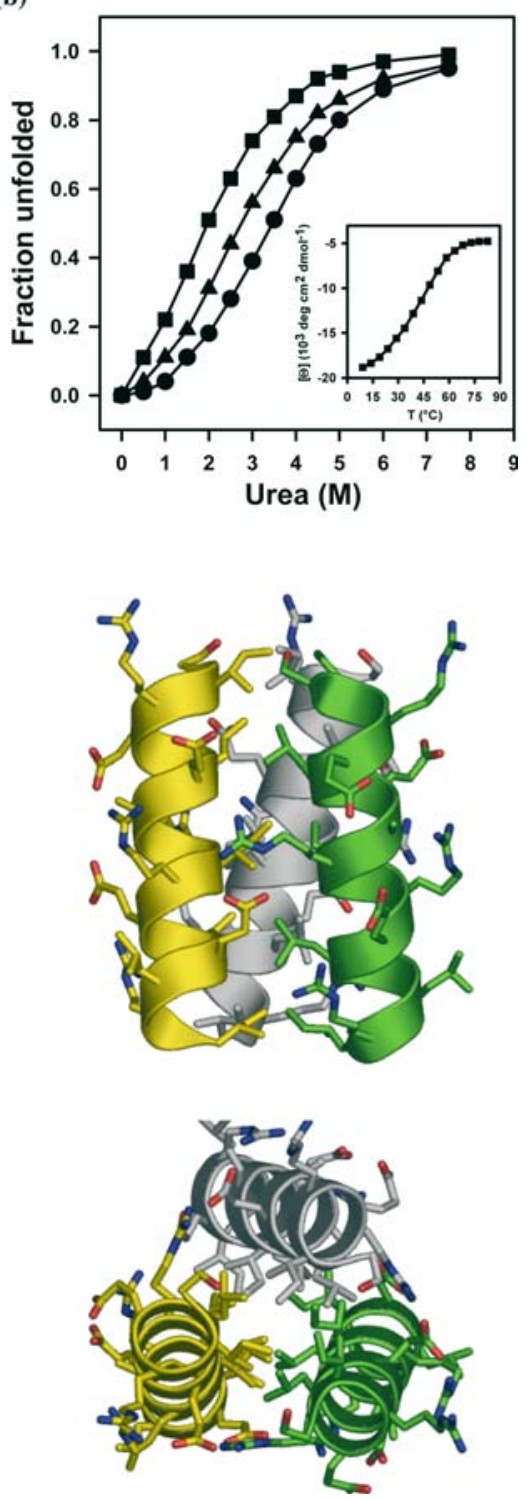

Fig. 2. $\operatorname{cc} \beta$ forms a stable native-like coiled-coil structure at low temperatures. (a), $\mathrm{CD}$ spectra recorded at $4^{\circ} \mathrm{C}$ from $0.2 \mathrm{mg} / \mathrm{ml} \mathrm{cc} \beta$-p $(\bullet), \operatorname{cc} \beta$ -

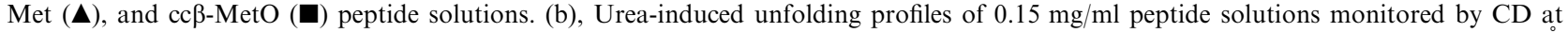
$222 \mathrm{~nm}$ and $4^{\circ} \mathrm{C}$. The symbols are the same as in A. All CD measurements were carried out in PBS. (c), Side and top views of the $2 \AA$ resolution crystal structure of the three-stranded cc $\beta$-p coiled coil (PDB ID code 1S9Z) in stereo. The monomer backbones and the residue side chains are represented as ribbons and sticks, respectively.

the monomeric peptides range between 2.1 and $2.2 \mathrm{kDa}$ ). The stabilities of $\operatorname{cc} \beta-\mathrm{p}$ and the two methionine variants were probed at $4^{\circ} \mathrm{C}$ by urea denaturation profiles recorded by $C D$ at $222 \mathrm{~nm}$ (Fig. 2b). At a peptide concentration of $0.15 \mathrm{mg} / \mathrm{ml}, \operatorname{cc} \beta-\mathrm{p}, \operatorname{cc} \beta$-Met and $\operatorname{cc} \beta$-MetO revealed sigmoidal denaturation profiles characteristic of a two-state helix-coil transition with single inflection points, $C_{\mathrm{m}}$, centered at $3.4,2.4$ and $1.5 \mathrm{M}$ urea, respectively.
The overall fold and topology of $\operatorname{cc} \beta-p$ in its $\alpha$-helical state was assessed by $\mathrm{X}$-ray crystallography. Crystals were obtained at $4^{\circ} \mathrm{C}$ and the structure was solved to a resolution of $2 \AA$ using the molecular replacement method (Kammerer et al. 2004). As shown in Figure 2c, $\operatorname{cc} \beta$-p forms a bundle of three parallel in-register $\alpha$-helices that wrap around each other in a slightly left-handed manner. The trimer has an average length of $27 \AA$ and an average diameter of $23 \AA$. 

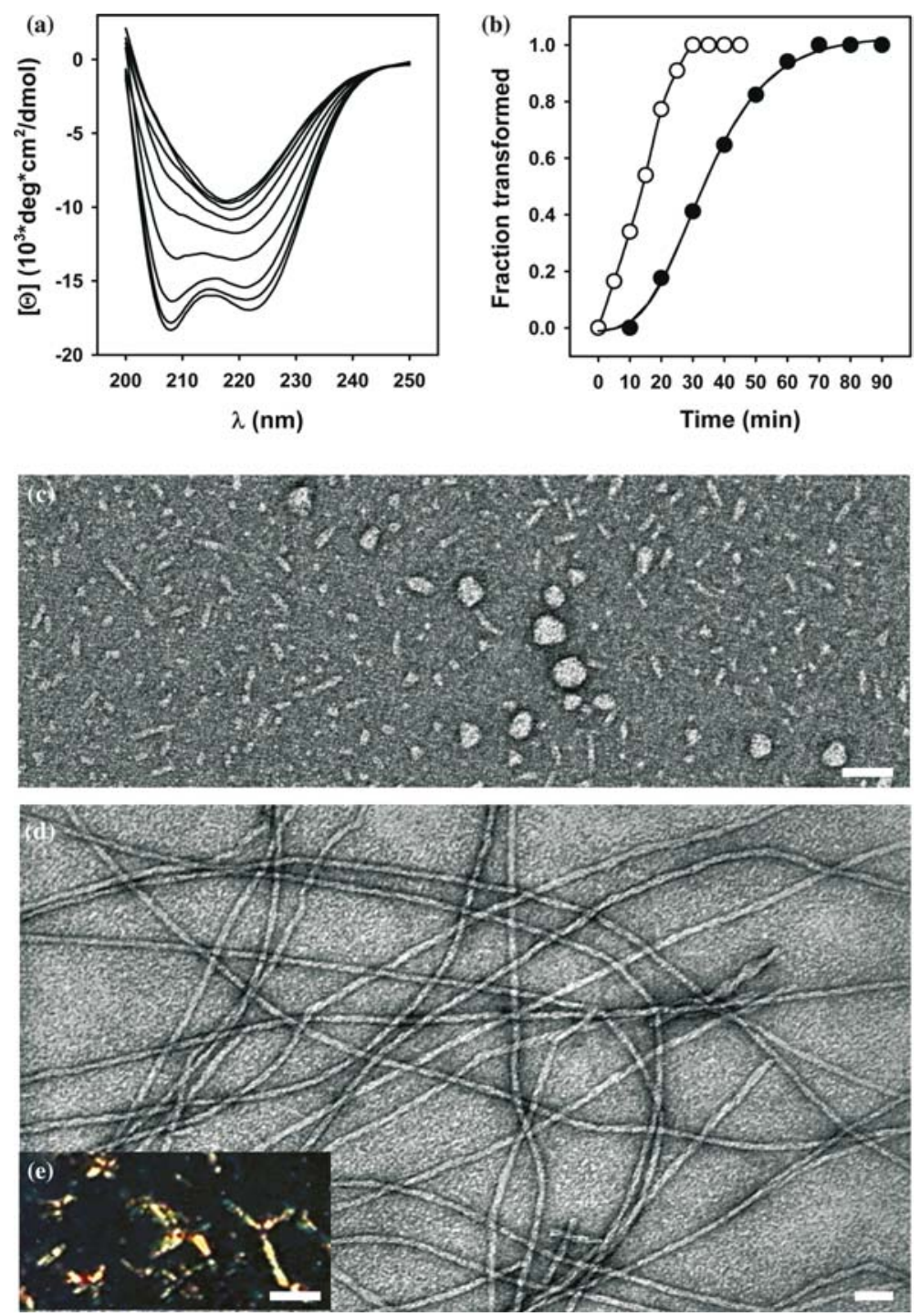

Fig. 3. $\operatorname{cc} \beta$ forms amyloid-like aggregates rich in $\beta$-sheet structure at elevated temperatures. (a), $\alpha$-to- $\beta$ transition of cc $\beta$-Met $(0.2 \mathrm{mg} / \mathrm{ml})$ monitored in PBS at $37^{\circ} \mathrm{C}$ by CD. The spectra were recorded in 10 min time intervals. (b), Time course of the $\alpha$-to- $\beta$ transition of cc $\beta$-Met $\left(0.2 \mathrm{mg} / \mathrm{ml}\right.$ in PBS) monitored by $\mathrm{CD}$ at $37^{\circ} \mathrm{C}$ and at $205 \mathrm{~nm}$ in the absence $(\mathbf{O})$ and presence $(O)$ of $5 \%(\mathrm{w} / \mathrm{w})$ preformed fibrils. (c), TEM micrograph of negatively stained cc $\beta$-p protofibrillar intermediates obtained in PBS. Scale bar, $50 \mathrm{~nm}$. (d), TEM micrograph of negatively stained mature cc $\beta$-p fibrils obtained in water. Scale bar, $50 \mathrm{~nm}$. (e), Congo red stained $\operatorname{cc} \beta$-p fibril sample obtained in PBS and imaged under cross-polarized light. Scale bar, $50 \mu \mathrm{m}$.

Consistent with a well-defined coiled-coil structure, the hydrophobic Ile and Leu side-chains occupying the a and $\mathbf{d}$ core positions are packed in a 'knobs-into-holes' fashion (Lupas, 1996). Arg8 and Glu13' form a tight belt of $\mathbf{g}$ to $\mathbf{e}^{\prime}$ interhelical salt bridges. Contrary to the intended design, none of the Glu and Arg residues form intrahelical salt-bridges but are mostly engaged in establishing crystal-packing contacts. The acetyl moiety of all three Serl residues was found to cap the helical monomers by forming an intrachain hydrogen bond with the $\mathrm{N}$-terminal main chain nitrogen atom.
It should be noted that trimer formation of $\operatorname{cc} \beta-p$ and its variants was unexpected and surprising because the peptides were originally designed to fold into two-stranded parallel coiled-coil structures (see above). This discrepancy suggests that in short coiledcoils residues at the $\mathbf{e}$ and $\mathbf{g}$ positions may play a more prominent role in specifying and stabilizing the oligomerization state than in longer structures.

The propensities of $\operatorname{cc} \beta-p, \operatorname{cc} \beta-$ Met, and $\operatorname{cc} \beta$ MetO to undergo a conformational switch was assessed by $\mathrm{CD}$ at elevated temperatures. Figure 3, a 
and $b$, shows the structural conversion of the cc $\beta$-Met coiled-coil. At $37^{\circ} \mathrm{C}$ and at a peptide concentration of $0.2 \mathrm{mg} / \mathrm{ml}$ the minima of the CD spectrum at 208 and $222 \mathrm{~nm}$ transformed within $1 \mathrm{~h}$ into a single minimum centered at $217 \mathrm{~nm}$ indicating a change in conformation from an $\alpha$-helix into a $\beta$-sheet-rich structure (Fig. 3a). After $2 \mathrm{~h}$, the CD signal at $217 \mathrm{~nm}$ started to decrease probably as a result of the formation of high molecular weight aggregates. No helical signal was regained upon cooling indicating that the heat-induced self-assembled $\beta$-sheet structures is irreversible. The change in structure was observed after a lag time of $\sim 12$ min (Fig. 3b). The length of the lag phase, as well as the slope of the growth phase, was temperature and concentration dependent (not shown). Addition of preformed aggregates rich in $\beta$-sheet structure shortened the transition time by eliminating the lag phase, and also accelerating the growth rate (Fig. 3b). These findings are consistent with a nucleation-dependent selfassembly process for the peptide (Rochet and Lansbury 2000). Unexpectedly, $\operatorname{cc} \beta-p$ aggregation was found to be $\sim 60$ times slower than that of the methionine peptide (not shown; (Kammerer et al. 2004)). In marked contrast to the aggregation behavior of $\operatorname{cc} \beta$-p and $\operatorname{cc} \beta$-Met, no aggregate formation was observed for the cc $\beta$-MetO derivative even after incubation of a $20 \mathrm{mg} / \mathrm{ml}$ peptide solution for $72 \mathrm{~h}$ at $90^{\circ} \mathrm{C}$ or after attempts to seed the system by addition of preformed cc $\beta$-Met fibrils. As shown in Fig. $2 b$ (inset), at a peptide concentration of $0.15 \mathrm{mg} /$ $\mathrm{ml}$ the thermal denaturation profile recorded from $\operatorname{cc} \beta$-MetO by CD at $222 \mathrm{~nm}$ revealed a fully reversible sigmoidal transition with a single midpoint of the unfolding transition, $T_{\mathrm{m}}$, centered at $46^{\circ} \mathrm{C}$.

The structure of $\operatorname{cc} \beta$-p and $\operatorname{cc} \beta$-Met in their $\beta$ sheet-rich form was analyzed by negative stain TEM. As shown in Figure 3c, peptide samples that were withdrawn during the early stages of assembly and inspected by TEM, revealed a series of discrete prefibrillar structures. These structures resemble the protofibrillar aggregates reported for a wide range of disease and non-disease related proteins (Walsh et al. 1999; Rochet and Lansbury 2000; Bucciantini et al. 2002). TEM micrographs of mature peptide samples revealed twisted and non-branched fibres 7 to $8 \mathrm{~nm}$ in diameter (Fig. 3d). The fibrils are strikingly similar to amyloid fibril assemblies reported for other peptides and proteins (Cohen et al. 1982). Furthermore, addition of Congo red to the sample produced a characteristic green birefringence under cross-polarized light (Fig. 3e). Importantly, detailed biophysical analyzes using Fourier-transform infrared spectroscopy, atomic force microscopy, scanning transmission electron microscopic mass-per-length measurements, X-ray fibre diffraction, and solid-state nuclear magnetic resonance were fully consistent with this conclusion. They provided solid support for a detailed molecular model for the peptide in its amyloid-like fibrillar state (Kammerer et al. 2004).

\section{CONCLUSIONS}

An essential requirement in the investigation of the relative contribution of factors such as sequence context, complementary side chain interactions, and protein stability to the formation of amyloid fibrils is the availability of well defined model systems. In this study, we used a minimalistic de novo design approach (Fig. 1) based on the coiled-coil structural motif to address these questions. Our solution and X-ray crystallographic data are consistent with the cc $\beta$ system forming a stable native-like coiled-coil structure (Fig. 2). Similarly, the data obtained on the aggregated state (Fig. 3 and ref. (Kammerer et al. 2004)) are consistent with well-ordered amyloid-like fibrils in which the peptide molecules are packed in a specific manner. These findings demonstrate that we have successfully designed a simple peptide sequence system that folds into a protein-like structure under ambient solution conditions and transforms into fibrils characteristic of amyloid structures at elevated temperatures.

A significant difference to other de novo model systems that form amyloid aggregates (Cerpa et al. 1996; Zhang and Rich 1997; Janek et al. 1999; West et al. 1999; Fezoui et al. 2000; Otzen et al. 2000; Takahashi et al. 2000; Ciani et al. 2002; Lopez De La et al. 2002; Tjernberg et al. 2002b) is that our designed 'miniprotein' adopts a stable tertiary fold despite its simplicity. This represents an important feature because it allows us to study the relationship between the stability of the native state of a protein and amyloid formation in a simplified manner. This is exemplified by the dramatic structural effects of a small chemical modification introduced by oxidizing methionine residues at selected hydrophobic positions. The insertion of only two oxygen atoms completely abolished amyloid fibril formation of the peptide while not significantly interfering with its coiled-coil structure. Importantly, these findings suggest that specific hydrophobic packing interactions can play a major role in the kinetics of amyloid formation (Kammerer et al. 2004). 
This conclusion may help to rationalize a number of pathological conditions linked to specific hydrophobic mutations that have little effect on the overall stability of the protein or on the other features mentioned above (Liemann and Glockshuber 1999; Chiba et al. 2003; Chiti et al. 2003). The characteristics of $\operatorname{cc} \beta$ make this model system thus highly suitable for probing molecular details of the assembly of amyloid structures (Miranker 2004). Its specific design together with the obtained detailed structural information now allows us to explore systematically both the effects of side chain packing interactions within the amyloid fibril and their relationship to the kinetics of amyloid fibril formation. This approach has an advantage over studies of natural disease-related models, where the complexity and size of the polypeptides often hinders a detailed rational approach to these processes.

\section{ACKNOWLEDGEMENTS}

We thank R. Wille for his excellent technical assistance and A. Lustig for performing the AUC experiments. R.A.K. is a Wellcome Senior Research Fellow in Basic Biomedical Science. M.O.S. was supported by Novartis Pharma AG during the initial phase of this work. This work was supported by a grant from the Swiss National Science Foundation (to MOS).

\section{REFERENCES}

Aguzzi, A. and Haass, C.: 2003, Science. 302, 814.

Albericio, F., Kneib-Cordonier, N., Biancalana, S., Gera, L., Masada, R. I., Hudson, D. and Barany, G.: 1990, J. Org. Chem. 55, 3730.

Balbirnie, M., Grothe, R. and Eisenberg, D. S.: 2001, Proc. Natl. Acad. Sci. U.S.A. 98, 2375.

Benzinger, T. L., Gregory, D. M., Burkoth, T. S., Miller-Auer, H., Lynn, D. G., Botto, R. E. and Meredith, S. C.: 1998, Proc. Natl. Acad. Sci. U.S.A. 95, 13407.

Booth, D. R., Sunde, M., Bellotti, V., Robinson, C. V., Hutchinson, W. L., Fraser, P. E., Hawkins, P. N., Dobson, C. M., Radford, S. E., Blake, C. C. and Pepys, M. B.: 1997, Nature. 385, 787.

Bucciantini, M., Giannoni, E., Chiti, F., Baroni, F., Formigli, L., Zurdo, J., Taddei, N., Ramponi, G., Dobson, C. M. and Stefani, M.: 2002, Nature. 416, 507.

Burke, M. G., Woscholski, R. and Yaliraki, S. N.: 2003, Proc. Natl. Acad. Sci. U.S.A. 100, 13928.

Burkhard, P., Meier, M. and Lustig, A.: 2000, Protein Sci. 9, 2294.

Burkhard, P., Stetefeld, J. and Strelkov, S. V.: 2001, Trends Cell Biol. 11, 82.

Cerpa, R., Cohen, F. E. and Kuntz, I. D.: 1996, Fold. Des. 1, 91. Chakrabartty, A. and Baldwin, R. L.: 1995, Adv. Protein Chem. 46, 141.
Chiba, T., Hagihara, Y., Higurashi, T., Hasegawa, K., Naiki, H. and Goto, Y.: 2003, J. Biol. Chem. 278, 47016.

Chiti, F., Webster, P., Taddei, N., Clark, A., Stefani, M., Ramponi, G. and Dobson, C. M.: 1999, Proc. Natl. Acad. Sci. U.S.A. 96,3590

Chiti, F., Taddei, N., Bucciantini, M., White, P., Ramponi, G. and Dobson, C. M.: 2000, EMBO J. 19, 1441.

Chiti, F., Taddei, N., Baroni, F., Capanni, C., Stefani, M., Ramponi, G. and Dobson, C. M.: 2002, Nat. Struct. Biol. 9, 137.

Chiti, F., Stefani, M., Taddei, N., Ramponi, G. and Dobson, C. M.: 2003, Nature. 424, 805.

Ciani, B., Hutchinson, E. G., Sessions, R. B. and Woolfson, D. N.: 2002, J. Biol. Chem. 277, 10150.

Cohen, C. and Parry, D. A.: 1990, Proteins. 7, 1.

Cohen, A. S., Shirahama, T. and Skinner, M.: 1982, Electron microscopy of amyloid. in J. R. Harris (ed.), Electron microscopy of proteins, Academic Press, London, UK.

Dado, P. G. and Gellman, S. H.: 1993, J. Am. Chem. Soc., 12609.

DeGrado, W. F., Summa, C. M., Pavone, V., Nastri, F. and Lombardi, A.: 1999, Annu. Rev. Biochem. 68, 779.

Fandrich, M., Fletcher, M. A. and Dobson, C. M.: 2001, Nature. $410,165$.

Fezoui, Y., Hartley, D. M., Walsh, D. M., Selkoe, D. J., Osterhout, J. J. and Teplow, D. B.: 2000, Nat. Struct. Biol. 7, 1095.

Frank, S., Lustig, A., Schulthess, T., Engel, J. and Kammerer, R. A.: 2000, J. Biol. Chem. 275, 11672.

García-Echeverría, C.: 1996, Bioorg. Med. Chem. Lett. 6, 229.

Hammarstrom, P., Jiang, X., Hurshman, A. R., Powers, E. T. and Kelly, J. W.: 2002, Proc. Natl. Acad. Sci. U.S.A. 99(4), 16427.

Harbury, P. B., Zhang, T., Kim, P. S. and Alber, T.: 1993, Science. 262, 1401.

Hecht, M. H., Das, A., Go, A., Bradley, L. H. and Wei, Y.: 2004, Protein Sci. 13, 1711.

Hurle, M. R., Helms, L. R., Li, L., Chan, W. and Wetzel, R.: 1994, Proc. Natl. Acad. Sci. U.S.A. 91, 5446.

Janek, K., Behlke, J., Zipper, J., Fabian, H., Georgalis, Y., Beyermann, M., Bienert, M. and Krause, E.: 1999, Biochemistry. 38, 8246.

Kammerer, R. A.: 1997, Matrix Biol. 15, 555.

Kammerer, R. A., Schulthess, T., Landwehr, R., Lustig, A., Engel, J., Aebi, U. and Steinmetz, M. O.: 1998, Proc. Natl. Acad. Sci. U.S.A. 95, 13419.

Kammerer, R. A., Jaravine, V. A., Frank, S., Schulthess, T., Landwehr, R., Lustig, A., Garcia-Echeverria, C., Alexandrescu, A. T., Engel, J. and Steinmetz, M. O.: 2001, J Biol. Chem. 276, 13685.

Kammerer, R. A., Kostrewa, D., Zurdo, J., Detken, A., GarciaEcheverria, C., Green, J. D., Muller, S. A., Meier, B. H., Winkler, F. K., Dobson, C. M. and Steinmetz, M. O.: 2004, Proc. Natl. Acad. Sci. U.S.A. 101, 4435.

Kelly, J. W. and Balch, W. E.: 2003, J. Cell Biol. 161, 461.

Kohn, W. D. and Hodges, R. S.: 1998, Trends Biotechnol. 16, 379.

Kyte, J. and Doolittle, R. F.: 1982, J Mol. Biol. 157, 105.

Lansbury, P. T. Jr.: 1999, Proc. Natl. Acad. Sci. U.S.A. 96, 3342.

Liemann, S. and Glockshuber, R.: 1999, Biochemistry. 38, 3258.

Lopez De La, P. M., Goldie, K., Zurdo, J., Lacroix, E., Dobson, C. M., Hoenger, A. and Serrano, L.: 2002, Proc. Natl. Acad. Sci. U.S.A. 99, 16052. 
Lu, M., Shu, W., Ji, H., Spek, E., Wang, L. and Kallenbach, N. R.: 1999, J Mol. Biol. 288, 743.

Lupas, A.: 1996, Trends Biochem. Sci. 21, 375.

McCutchen, S. L., Lai, Z., Miroy, G. J., Kelly, J. W. and Colon, W.: 1995, Biochemistry. 34, 13527.

Minor, D. L. Jr. and Kim, P. S.: 1994, Nature. 367, 660.

Miranker, A. D.: 2004, Proc. Natl. Acad. Sci. U.S.A. 101, 4335.

Nielsen, L., Frokjaer, S., Brange, J., Uversky, V. N. and Fink, A. L.: 2001, Biochemistry. 40, 8397.

Otzen, D. E., Kristensen, O. and Oliveberg, M.: 2000, Proc. Natl. Acad. Sci. U.S.A. 97, 9907.

Perutz, M. F.: 1999, Trends Biochem. Sci. 24, 58.

Perutz, M. F., Johnson, T., Suzuki, M. and Finch, J. T.: 1994, Proc. Natl. Acad. Sci. U.S.A. 91, 5355.

Prusiner, S. B.: 2001, N. Engl. J. Med. 344, 1516.

Ramirez-Alvarado, M., Merkel, J. S. and Regan, L.: 2000, Proc. Natl. Acad. Sci. U.S.A. 97, 8979.

Richardson, J. S. and Richardson, D. C.: 1998, Science. 240, 1648.

Rochet, J. C. and Lansbury, P. T. Jr: 2000, Curr. Opin. Struct. Biol. 10,60 .

Sacchettini, J. C. and Kelly, J. W.: 2002, Nat. Rev. Drug Discov. $1,267$.

Serpell, L. C., Sunde, M. and Blake, C. C.: 1997, Cell Mol. Life Sci. 53, 871.

Smith, D. P., Jones, S., Serpell, L. C., Sunde, M. and Radford, S. E.: 2003, J. Mol. Biol. 330, 943.

Stefani, M. and Dobson, C. M.: 2003, J. Mol. Med. 81, 678.

Steinmetz, M. O., Stock, A., Schulthess, T., Landwehr, R., Lustig, A., Faix, J., Gerisch, G., Aebi, U. and Kammerer, R. A.: 1998, EMBO J. 17, 1883.

Su, J. Y., Hodges, R. S. and Kay, C. M.: 1994, Biochemistry. 33, 15501.
Sunde, M., Serpell, L. C., Bartlam, M., Fraser, P. E., Pepys, M. B. and Blake, C. C.: 1997, J. Mol. Biol. 273, 729.

Takahashi, Y., Ueno, A. and Mihara, H.: 2000, Structure. Fold. Des. 8, 915.

Taylor, J. P., Hardy, J. and Fischbeck, K. H.: 2002, Science. 296, 1991.

Tenidis, K., Waldner, M., Bernhagen, J., Fischle, W., Bergmann, M., Weber, M., Merkle, M. L., Voelter, W., Brunner, H. and Kapurniotu, A.: 2000, J. Mol. Biol. 295, 1055.

Tjernberg, L. O., Callaway, D. J., Tjernberg, A., Hahne, S., Lilliehook, C., Terenius, L., Thyberg, J. and Nordstedt, C.: 1999, J. Biol. Chem. 274, 12619.

Tjernberg, L., Hosia, W., Bark, N., Thyberg, J. and Johansson, J.: 2002a, J. Biol. Chem. 277, 43243.

Tjernberg, L. O., Tjernberg, A., Bark, N., Shi, Y., Ruzsicska, B. P., Bu, Z., Thyberg, J. and Callaway, D. J.: 2002b, Biochem. J. $366,343$.

Villegas, V., Zurdo, J., Filimonov, V. V., Aviles, F. X., Dobson, C. M. and Serrano, L.: 2000, Protein Sci. 9, 1700.

Walsh, D. M., Hartley, D. M., Kusumoto, Y., Fezoui, Y., Condron, M. M., Lomakin, A., Benedek, G. B., Selkoe, D. J. and Teplow, D. B.: 1999, J. Biol. Chem. 274, 25945.

West, M. W., Wang, W., Patterson, J., Mancias, J. D., Beasley, J. R. and Hecht, M. H.: 1999, Proc. Natl. Acad. Sci. U.S.A. 96, 11211.

Zhang, S.: 2003, Nat. Biotechnol. 21, 1171.

Zhang, S. and Rich, A.: 1997, Proc. Natl. Acad. Sci. U.S.A. 94, 23.

Zhu, L., Zhang, X. J., Wang, L. Y., Zhou, J. M. and Perrett, S.: 2003, J. Mol. Biol. 328, 235. 